\title{
Relato de experiência sobre intervenção educacional na Atenção Básica para prevenção da sífilis na gestação ressaltando sua influência nas complicações materno-
}

\section{fetais}

\author{
Experience report on educational intervention in Primary Care for the prevention of syphilis \\ during pregnancy standing out its influence on maternal-fetal complications \\ Informe de experiencia sobre intervención educativa en Atención Primaria para la prevención de la \\ sífilis durante el embarazo, destacando su influencia en las complicaciones materno-fetales
}

Ana Karolyne Monteiro da Silva ORCID: https://orcid.org/0000-0002-5955-9306

Universidade Ceuma, Brasil

E-mail: ana90651@ceuma.com.br

Amanda Rafaella Garcia Avelino ORCID: https://orcid.org/0000-0002-4319-4488 Universidade Ceuma, Brasil E-mail: amandaravelino@gmail.com

Keven Rufino Menezes

ORCID: https://orcid.org/0000-0001-8831-4220

Universidade Ceuma, Brasil

E-mail: kevi.imp@gmail.com

Rafael Abutrab Souza Ramos Silva ORCID: https://orcid.org/0000-0002-7893-6613 Universidade Ceuma, Brasil

E-mail: rafaelabutrab2014@outlook.com

Ricardo Freitas de Oliveira ORCID: https://orcid.org/0000-0001-9958-1500 Universidade Ceuma, Brasil

E-mail: ricardo_freitasoliver@hotmail.com

Paulo da Costa Araújo ORCID: https://orcid.org/0000-0001-5106-8505 Universidade Ceuma, Brasil E-mail: paulo7ca@gmail.com

Hygor Regadas Barros Souza ORCID: https://orcid.org/0000-0001-9542-7047 Universidade Ceuma, Brasil E-mail: hygorbarros34@gmail.com

Antonio Gustavo da Mota Goveia ORCID: https://orcid.org/0000-0002-0702-6217 Universidade Ceuma, Brasil

E-mail: antoniogustavo_22@hotmail.com

Maria Gabryela Oliveira Costa ORCID: https://orcid.org/0000-0003-3733-4373 Universidade Ceuma, Brasil

E-mail: mariagaby44@hotmail.com

Ana Clara Pimentel Lima ORCID: https://orcid.org/0000-0001-6264-7641 Universidade Ceuma, Brasil

E-mail: ac_pimentell@ hotmail.com

Yasmin de Matos Capistranio ORCID: https://orcid.org/0000-0003-2087-674X Universidade Ceuma, Brasil

E-mail: yasmincapistranio14@gmail.com

Janine Silva Ribeiro Godoy ORCID: https://orcid.org/0000-0001-5587-0896 Universidade Ceuma, Brasil E-mail: janine.silva@ ceuma.br 


\begin{abstract}
Resumo
O estudo teve como objetivo relatar a experiência de acadêmicos de Medicina na realização de atividades de educação em saúde para demonstrar a incidência da Sífilis gestacional em nosso meio e, dessa forma, permitir a reflexão sobre o assunto e propor estratégias para que esses casos se reduzam, melhorando a qualidade de vida da gestante e impedindo a transmissão para o feto. As atividades foram realizadas em uma Unidade Básica de Saúde no Bairro Santa Rita, na cidade de Imperatriz-MA. A ação foi dividida em três etapas: a $1^{\mathrm{a}}$ etapa consistiu em uma breve apresentação de todos; a $2^{\mathrm{a}}$ etapa uma roda de conversa na qual foram abordados temas referentes à sífilis; a $3^{\mathrm{a}}$ etapa constituiu-se na aplicação de um questionário para avaliação do conhecimento e satisfação das participantes. Foram observadas as dificuldades das gestantes em apreender e memorizar as informações que receberam durante as consultas de pré-natal. Estas relataram, ainda, não saberem que a infecção poderia ser transmitida ao feto, nem que há a necessidade de se tratar concomitantemente o parceiro. Conclui-se que a gestante, em posse das informações necessárias, se torna totalmente capaz de evitar contaminação própria e do bebê, atua também como agente disseminadora do conhecimento para o parceiro e outras gestantes sobre: os sinais e sintomas, a necessidade da realização do teste não treponêmico VDRL ainda durante o período gestacional e do tratamento adequado para prevenir que a criança venha a ser infectada.
\end{abstract}

Palavras-chave: Sífilis gestacional; Congênita; Transmissão.

\begin{abstract}
The study aimed to report an experience of medical students in carrying out health education activities to demonstrate the increase in gestational syphilis in our country and, thus, allow a reflection on the subject and proportions so that these cases are reduced, improving the quality of life of pregnant women and preventing transmission to the fetus. The activities were carried out in a Basic Health Unit in Bairro Santa Rita, in the city of Imperatriz-MA. The action was divided into three stages: the 1st stage consisted of a brief presentation by everyone; a 2nd stage, a conversation circle in which syphilis themes were defined; the 3rd stage consisted of applying a questionnaire to assess participants' knowledge and satisfaction. The difficulties of pregnant women to grasp and memorize the information they received during prenatal consultations were observed. They also reported not knowing that the infection could be transmitted to the fetus, nor that there is a need to treat the partner at the same time. It is concluded that a pregnant woman, in possession of the information, becomes fully capable of avoiding the contamination of herself and the baby, as well as a dissemination agent of knowledge to the partner and other pregnant women about: signs and symptoms, the need for realization the non-treponemal VDRL test during the gestational period and the adequate treatment to prevent the child from becoming infected.
\end{abstract}

Keywords: Gestational syphilis; Congenital; Transmission.

\title{
Resumen
}

El estudio tuvo como objetivo reportar la experiencia de estudiantes de medicina en la realización de actividades de educación en salud para demostrar la incidencia de la sífilis gestacional en nuestro país y, así, permitir la reflexión sobre el tema y proponer estrategias para reducir estos casos, mejorando la calidad de vida de las gestantes. mujeres y prevenir la transmisión al feto. Las actividades se realizaron en una Unidad Básica de Salud del Barrio Santa Rita, en la ciudad de Imperatriz-MA. La acción se dividió en tres etapas: la $1^{\text {a }}$ etapa consistió en una breve presentación de todos; la $2^{\mathrm{a}}$ etapa un círculo de conversación en el que se abordaron temas relacionados con la sífilis; la $3^{\mathrm{a}}$ etapa consistió en la aplicación de un cuestionario para evaluar el conocimiento y la satisfacción de los participantes. Se observó la dificultad de las mujeres embarazadas para captar y memorizar la información recibida durante las consultas prenatales. También informaron que no sabían que la infección podría transmitirse al feto, ni que era necesario tratar a la pareja al mismo tiempo. Se concluye que la gestante, en posesión de la información necesaria, se vuelve plenamente capaz de evitar la contaminación de sí misma y del bebé, además actúa como agente de difusión del conocimiento para la pareja y otras gestantes sobre: los signos y síntomas, la necesidad de realizar la prueba VDRL no treponémica durante el período gestacional y tratamiento adecuado para evitar que el niño se infecte.

Palabras clave: Sífilis gestacional; Congénita; Transmisión.

\section{Introdução}

O presente trabalho se trata de um relato de prática sobre a prevenção de sífilis gestacional no bairro Santa Rita, localizado na cidade de Imperatriz, no estado do Maranhão. O objetivo deste relato é demonstrar a incidência dessa doença em nosso meio e, dessa forma, permitir a reflexão sobre o assunto e propor estratégias para que esses casos se reduzam, melhorando a qualidade de vida da gestante e impedindo a transmissão para o feto.

A sífilis, doença sexualmente transmitida causada pela bactéria Treponema pallidum, gênero Treponema, da família dos Treponemataceae, se apresenta como um desafio à saúde pública em todo o mundo. É uma doença transmitida por via 
sexual e vertical, pela placenta da mãe para o feto. Outras formas de transmissão podem ser por via indireta (objetos contaminados) e por transfusão sanguínea (Damasceno et al, 2014).

Existem dois tipos de sífilis: a congênita e a adquirida. A congênita é transmitida verticalmente pela disseminação transplacentária, ao passo que a adquirida, é sexualmente transmitida. A doença adquirida pode ser classificada em quatro estágios: primário, secundário, latente e terciário (Fonseca, 2017).

A sífilis congênita é decorrente da disseminação hematogênica do agente etiológico Treponema pallidum da gestante não tratada ou inadequadamente tratada para o seu concepto, por via transplacentária. A transmissão pode ocorrer em qualquer fase da gestação e em qualquer estágio da doença, com probabilidades de 50\% a 100\% na sífilis primária e secundária, 40\% na sífilis latente precoce e $10 \%$ na sífilis latente tardia. É possível transmissão direta no canal do parto. Ocorrendo a transmissão da sífilis congênita, cerca de $40 \%$ dos casos podem evoluir para aborto espontâneo, natimorto e óbito perinatal (Epidemiológica, 2008).

Essa comorbidade é dividida em dois períodos: a precoce (até o segundo ano de vida) e a tardia (surge após segundo ano de vida). A maior parte dos casos de sífilis congênita precoce é assintomática (cerca de 70\%), porém o recém-nascido pode apresentar prematuridade, baixo peso, hepatomegalia, esplenomegalia, lesões cutâneas (pênfigo sifilítico, condiloma plano, petéquias, púrpura, fissura peribucal), periostite, osteocondrite, pseudoparalisia dos membros, sofrimento respiratório com ou sem pneumonia, rinite sero-sanguinolenta, icterícia, anemia, linfadenopatia generalizada, síndrome nefrótica, convulsão e meningite, trombocitopenia, leucocitose ou leucopenia. Na sífilis congênita tardia, as manifestações clínicas são raras e resultantes da cicatrização da doença sistêmica precoce, podendo envolver vários órgãos (Epidemiológica, 2008).

É um contrassenso uma doença de fácil diagnóstico e terapêutica medicamentosa de tão baixo custo apresentar incidência tão elevada na nossa população. As consequências são desastrosas, principalmente para as pacientes em idade reprodutiva, pelos riscos de transmissão vertical nos casos de sífilis congênita. Sendo assim, é de suma importância uma intervenção que poderá ser útil para melhor entendimento e aplicabilidade de políticas de saúde que ajudariam no combate à doença (Damasceno, 2014).

Segundo estimativas da Organização Mundial da Saúde (OMS), ocorrem 340 milhões de casos de infecções sexualmente transmissíveis (IST) no mundo por ano, entre as quais 12 milhões são de sífilis e, em 90\% dos casos, acontece em países em desenvolvimento. A doença na gestante é um agravo de notificação compulsória para fins de vigilância epidemiológica desde 2005 e estima-se que apenas 32\% dos casos são notificados, refletindo uma importante deficiência na qualidade dos serviços de assistência ao pré-natal e ao parto (Magalhães, et al, 2011).

A não realização de pré-natal, a gravidez na adolescência, o uso de drogas ilícitas pela mãe ou pelo parceiro (principalmente crack/cocaína), a ausência de parceiro sexual fixo e/ou a existência de múltiplos parceiros, baixa escolaridade e nível socioeconômico, multiparidade, acesso limitado aos serviços de saúde e presença de outras infecções sexualmente transmissíveis (IST's) na mulher ou no parceiro são fatores de risco associados à infecção congênita (Damasceno, 2014).

O bom acompanhamento gestacional reduz bastante os riscos de complicações durante a gestação. Além disso, essa assistência bem realizada permite uma boa atuação da equipe da sala de parto, permitindo a diminuição de riscos, infecções e problemas no pós-parto. Logo, melhora a qualidade de vida do núcleo familiar (Rocha, 2010).

A assistência ao pré-natal constitui um meio em favor da mulher grávida e do concepto. Esta atenção inicia-se desde a concepção, sendo então preventiva, até o início do trabalho de parto, tendo como objetivos identificar, tratar ou controlar doenças, prevenir complicações na gestação e parto, reduzir os índices de morbimortalidade materna e fetal (Netto, 2021).

Dessa forma, é possível fazer ações educativas, com esclarecimento, ressaltando a importância de um bom acompanhamento pré-natal para as gestantes cadastradas na área em momentos que as pacientes aguardam atendimento, por 
meio de rodas de conversas, onde se possa discutir as principais prevenções, a importância das consultas, o uso de determinadas medicações e sobre infecções sexualmente transmissíveis (Damasceno, 2014).

\section{Metodologia}

Trata-se de um estudo descritivo, exploratório, do tipo relato de experiência, desenvolvido com um grupo de gestantes em uma Unidade Básica de Saúde (UBS) no bairro Santa Rita na cidade de Imperatriz-MA. Os relatos de experiência são tidos como metodologias de observação sistemática da realidade, sem o objetivo de testar hipóteses, mas estabelecendo uma relação entre os achados dessa realidade e as bases teóricas pertinentes. O grupo apresenta gestantes em faixa etária bastante diversificada, sendo que a mais jovem apresentava 15 anos e a mais velha encontrava-se na faixa dos 36 anos. O encontro foi realizado em um dia, na sala de triagem da UBS. Para a realização da ação descrita nesse trabalho, foi agendado previamente o encontro dos acadêmicos com o grupo por intermédio do enfermeiro e do médico da unidade. Na data marcada compareceram 15 gestantes. No estudo, foram respeitados aos princípios bioéticos preconizados na Resolução 466/12 do Conselho Nacional de Saúde.

\section{Resultados e Discussão}

O planejamento da ação foi realizado durante o semestre 2021.2, sob a orientação da professora Dra. Janine Silva Ribeiro Godoy. Foi realizado com o apoio dos profissionais da Unidade Básica de Saúde Santa Rita, que forneceu a sala de reuniões e divulgou nossa ação naquela instituição.

A ação foi dividida em três etapas, o primeiro momento consistiu em uma breve apresentação da equipe que iria direcionar as ações (Figura 1) e, posteriormente, o grupo de gestantes também foi convidado a apresentar-se, a fim de que fosse criada uma familiarização logo no início da oficina.

Na segunda etapa, constituiu-se a roda de conversa na qual foram abordados temas referentes à sífilis, tais como: sífilis na gestação, consequências da infecção para o binômio mãe-filho, modo de transmissão, prevenção e tratamento. Nesse momento, foram utilizadas imagens e desenhos referente aos sinais e sintomas, representando o quadro clínico da doença, permitindo intervenções e questionamentos por parte das participantes (Figura 2). Além disso, foi entregue a elas, um panfleto abordando tudo sobre a sífilis, de forma resumida, com um conteúdo criativo e informativo (Figura 3).

Observou-se a dificuldades das gestantes em apreender e memorizar as informações que receberam durante as consultas de pré-natal. Este fato foi evidenciado por relatos daquelas ao afirmarem que já haviam ouvido falar da doença, porém não sabiam ou não lembravam seus sintomas, mecanismos de prevenção ou tratamento. As participantes relataram não terem conhecimento que a infecção poderia ser transmitida ao feto, nem que há a necessidade de se tratar concomitantemente o parceiro.

Assim, percebe-se que as gestantes não apreenderam sobre transmissão e tratamento da sífilis durante o pré-natal. Entende-se, portanto, que a maneira mais concreta de alcançar a prevenção e o controle da sífilis congênita está no compromisso da atenção básica de oferecer as gestantes uma assistência de pré-natal de qualidade, garantindo um diagnóstico precoce e tratamento adequado para evitar transmissão vertical da doença.

As literaturas afirmam que embora a sífilis seja uma doença para a qual existem recursos diagnósticos e terapêuticos simples e de e de baixo custo, seu controle na gestação mostra-se um desafio para profissionais de saúde e gestores. Isso em decorrência do curto intervalo da gestação para a realização do seu diagnóstico e tratamento; pela dificuldade de abordagem das doenças sexualmente transmissíveis, principalmente durante a gestação; e, provavelmente, pelo desconhecimento da 
magnitude desse agravo e dos danos que ele poderá causar à saúde da mulher e do bebê pela população e pelos profissionais de saúde.

Com isso, a etapa final permitiu analisar o nível de conhecimento adquirido pelas gestante, por meio da aplicação de um questionário em que as participantes responderam com "sim, não ou indiferente" (Figura 4), a pesquisa se mostrou bastante favorável, visto que a maior parte das perguntas foram respondidas positivamente, demonstrando que o objetivo da intervenção foi atingido e que proporcionou promoção de saúde com esse grupo (Figura 5).

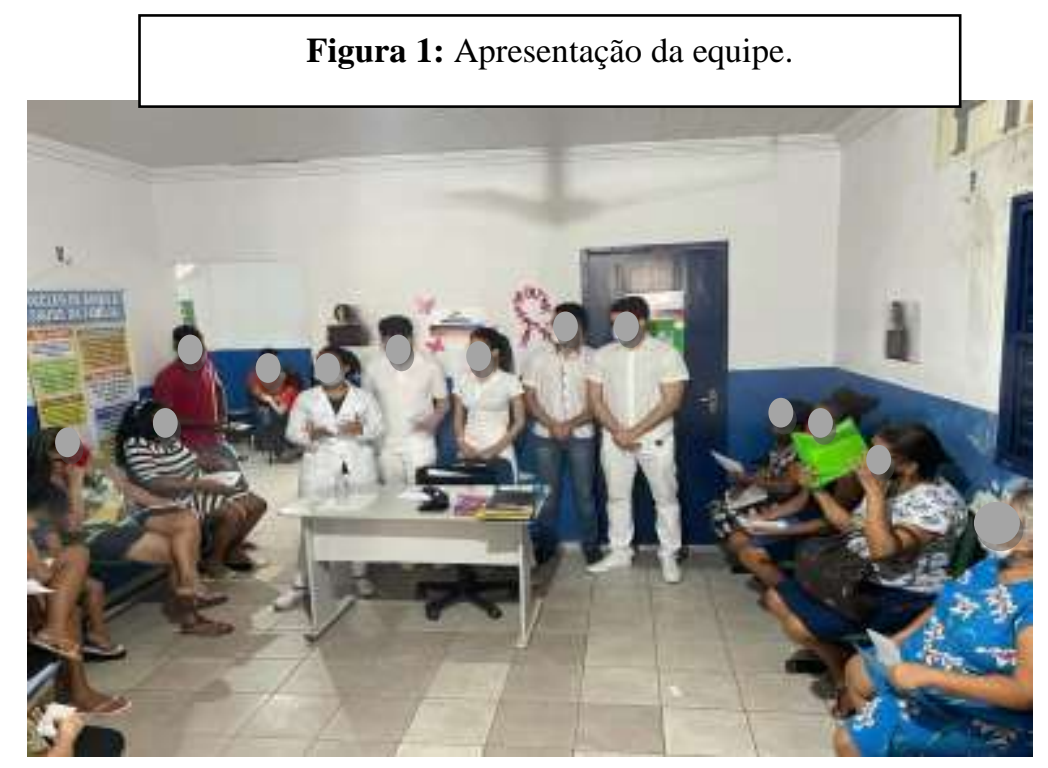

Fonte: Unidade Básica de Saúde Santa Rita.

Figura 2: Conversa com gestante, mostrando imagens sobre os principais sinais do quadro clínico da sífilis.

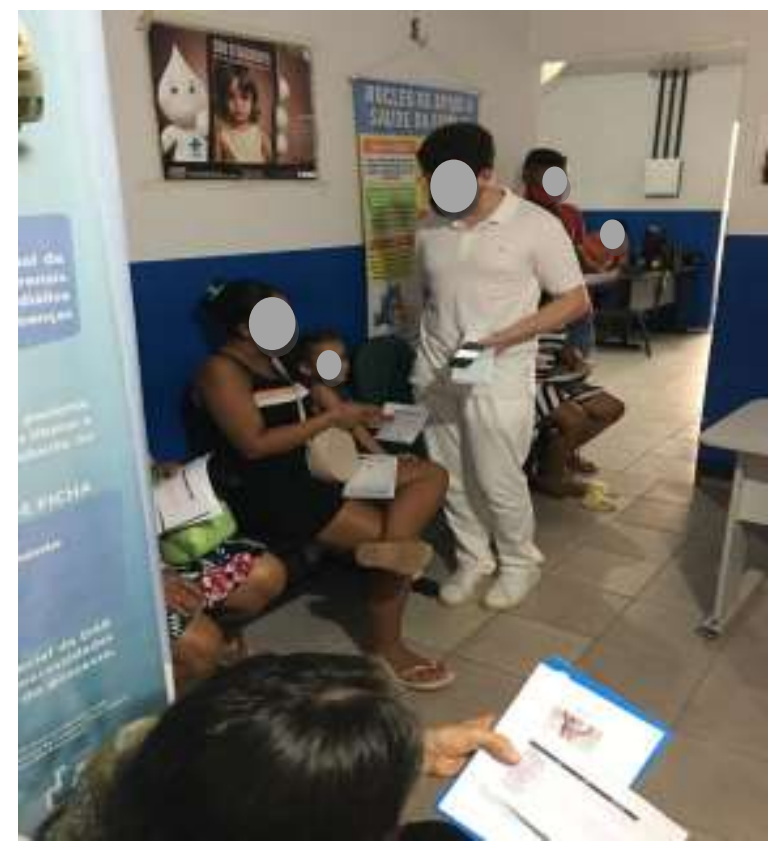

Fonte: Unidade Básica de Saúde Santa Rita. 
Research, Society and Development, v. 11, n. 2, e15611225501, 2022

(CC BY 4.0) | ISSN 2525-3409 | DOI: http://dx.doi.org/10.33448/rsd-v11i2.25501

Figura 3: Capa do panfleto entregue aos participantes.

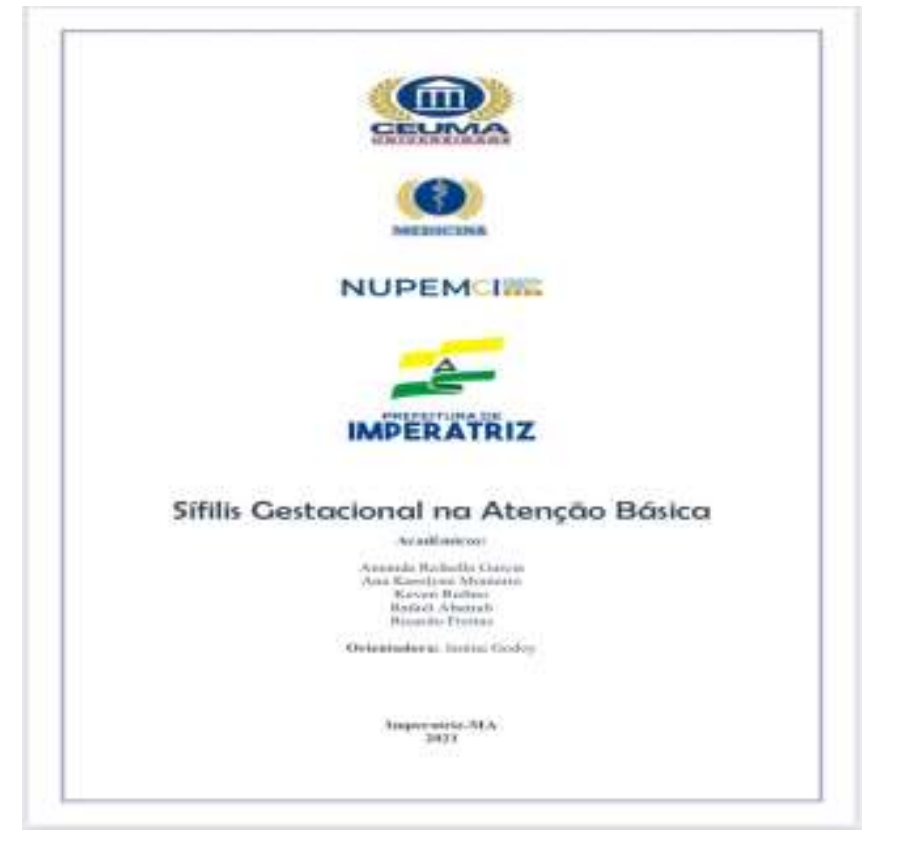

Fonte: Unidade Básica de Saúde Santa Rita.

Figura 4: Questionário aplicado.

\begin{tabular}{|l|l|l|}
\hline \multicolumn{1}{|c|}{ Sim } & Nä̀ & Indiferente \\
\hline $\begin{array}{l}\text { O evento } \\
\text { correspondeu às }\end{array}$ & & \\
suas expectativas? & & \\
\hline $\begin{array}{l}\text { Você conseguiu tirar } \\
\text { suas dúvidas sobre o } \\
\text { tema abordado? }\end{array}$ & & \\
\hline $\begin{array}{l}\text { O que foi ministrado } \\
\text { vai interferir }\end{array}$ & & \\
positivamente no & & \\
seu pré-natal? & & \\
\hline $\begin{array}{l}\text { Você considera o } \\
\text { tema relevante para }\end{array}$ & & \\
a saúde pública? & & \\
\hline
\end{tabular}

Fonte: Unidade Básica de Saúde Santa Rita. 


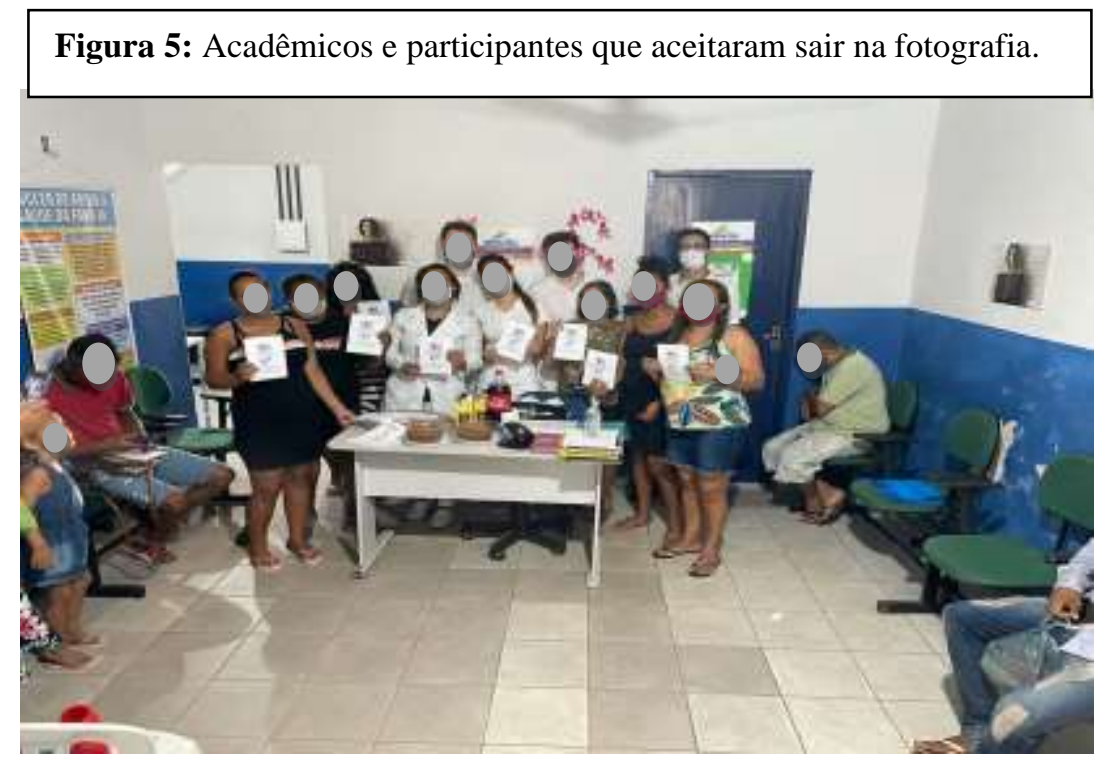

Fonte: Unidade Básica de Saúde Santa Rita.

\section{Considerações Finais}

Constatou-se que esse momento de educação em saúde proporcionou um aumento do conhecimento sobre sífilis em gestantes, além disso, relacionou que a prevenção da doença também consiste em uma forma de manifestar cuidado com a evolução da gestação.

A educação em saúde demonstra ser uma importante ferramenta para os profissionais no que se refere à prevenção de doenças e promoção da saúde. No caso especial da sífilis, a gestante, em posse das informações necessárias, se torna totalmente capaz de evitar contaminação própria e do bebê, atua também como agente disseminadora do conhecimento para o parceiro e outras gestantes sobre: os sinais e sintomas, a necessidade da realização do teste não treponêmico VDRL ainda durante o período gestacional e do tratamento adequado para prevenir que a criança venha a ser infectada.

Este relato de experiência esperar-se servir como base para intervenções na área. Ressalta-se que não se constitui como um modelo único e completo, recomendada a adaptação a cada contexto. Ademais, espera-se que este trabalho leve a outras discussões e pesquisas em relação a sífilis gestacional e sua abordagem na Atenção Básica.

\section{Referências}

Almeida, K. C. D., Lindolfo, L. C., \& Alcântara, K. C. D. (2009). Sífilis em gestantes atendidas em uma unidade de saúde pública de Anápolis, Goiás, Brasil. Rev. bras. anal. clin, 181-184.

Bottura, B. R., Matuda, L., Rodrigues, P. S. S., do Amaral, C. M. C. A., \& Barbosa, L. G. (2019). Perfil epidemiológico da sífilis gestacional e congênita no Brasil-período de 2007 a 2016/Epidemiological profile of gestational and congenital syphilis in Brazil-from 2007 to 2016. Arquivos Médicos dos Hospitais e da Faculdade de Ciências Médicas da Santa Casa de São Paulo, 64(2), 69-75.

Brasil (2008). Fundação Nacional de Saúde (Funasa). Diretrizes de educação em saúde visando à promoção da saúde: documento base - documento I: Brasília. Brasil (2006). Ministério da Saúde (MS). Secretaria de Vigilância em Saúde. Programa Nacional de DST/AIDS. Diretrizes para controle da sífilis congênita: manual de bolso. (2a ed.).

Brasil (2021). Boletim Epidemiológico. Secretaria de Vigilância em Saúde Ministério da Saúde. Editora MS/CGDI.

Cabral, B. T. V., da Costa Dantas, J., da Silva, J. A., \& de Oliveira, D. A. (2017). Sífilis em gestante e sífilis congênita: um estudo retrospectivo. Revista ciência plural, 3(3), 32-44.

Costa, Joávio Soares et al (2016). O conhecimento de gestantes com diagnóstico de sífilis sobre a doença. Revista interdisciplinar, 9(2), 79-89.

Costa, C. C. D., Gomes, L. F. D. S., Teles, L. M. R., Mendes, I. C., Oriá, M. O. B., \& Damascen, A. K. D. C. (2020). Construção e validação de uma tecnologia educacional para prevenção da sífilis congênita. Acta Paulista de Enfermagem, 33. 
da Fonseca Wastner, B., Melzer, R. S., do Nascimento, T. C. D. L., Parise, G. K., Stramandinoli-Zanicotti, R. T., \& Sassi, L. M. (2017). Sífilis secundária em gestante: relato de caso. Archives Of Health Investigation, 6(9).

Damasceno, A. B., Monteiro, D. L., Rodrigues, L. B., Barmpas, D. B. S., Cerqueira, L. R., \& Trajano, A. J. (2014). Sífilis na gravidez. Revista Hospital Universitário Pedro Ernesto, 13(3).

Domingues, R. M. S. M., Saracen, V., Hartz, Z. M. D. A., \& Leal, M. D. C. (2013). Sífilis congénita: evento centinela de la calidad de la asistencia prenatal. Revista de Saúde pública, 47(1), 147-157.

da Silva, J. G., Gomes, G. C., Ribeiro, J. P., de Jung, B. C., de Oliveira Nörberg, P. K., \& Mota, M. S. (2019). Sífilis gestacional: repercussões para a puérpera. Cogitare Enfermagem, 24.

de Freitas Silva, M. F. C., Pereira, S. M. X., Aidar, T. D. P. S., de Souza, R. G., de Carvalho Costa, R. F., de Oliveira, L. A. G., \& Dantas, P. C. (2020). Sífilis congênita como uma abordagem sistêmica. Brazilian Journal of Development, 6(7), 51840-51848.

de Almeida Lemos, L. S. C., de Souza, G. S., Vasconcelos, B. M., Gama, C. R., de Medeiros Silva, L. S., Cerqueira, T. M. G., \& da Silva Noberto, D. (2019). O pré-natal como ferramenta na prevenção da sífilis congênita: uma revisão integrativa da literatura. Brazilian Journal of Health Review, 2(3), $1616-1623$.

EPIDEMIOLÓGICA, S. D. V. (2008). Sífilis congênita e sífilis na gestação. Revista Saúde Pública, 42(4), 768-72.

Figueiredo, D. C. M. M. D., Figueiredo, A. M. D., Souza, T. K. B. D., Tavares, G., \& Vianna, R. P. D. T. (2020). Relação entre oferta de diagnóstico e tratamento da sífilis na atenção básica sobre a incidência de sífilis gestacional e congênita. Cadernos de Saúde Pública, 36.

Guimarães, T. A., Alencar, L. C. R., Fonseca, L. M. B., Gonçalves, M. M. C., \& da Silva, M. P. (2018). Sífilis em gestantes e sífilis congênita no Maranhão. Arquivos de Ciências da Saúde, 25(2), 24-30.

Lazarini, F. M, \& Barbosa, D. A (2017). Intervenção educacional na Atenção Básica para prevenção da sífilis congênita1. Revista Latino-Americana de Enfermagem, 25.

Luo, Y., Xie, Y., \& Xiao, Y. (2021). Ferramentas de diagnóstico laboratorial para sífilis: situação atual e perspectivas futuras. Fronteiras em microbiologia celular e de infecção, 896.

Magalhães, D. M. D. S., Kawaguchi, I. A. L., Dias, A., \& Calderon, I. D. M. P. (2013). Sífilis materna e congênita: ainda um desafio. Cadernos de Saúde Pública, 29, 1109-1120.

Mesquita, K. O., Lima, G. K., Filgueira, A. A., Flôr, S. M. C., Freitas, C. A. S. L., Linhares, M. S. C., \& Gubert, F. A. (2012). Análise dos casos de sífilis congênita em Sobral, Ceará: contribuições para assistência pré-natal. DST-J Bras Doenças Sex Transm, 24(1), 20-7.

Milanez, H., \& Amaral, E. (2008). Por que ainda não conseguimos controlar o problema da sífilis em gestantes e recém-nascidos? Revista Brasileira de Ginecologia e Obstetrícia, 30(7), 325-327.

Netto, J. L. D. M. G., da Silva, A. P., Medeiros, E. B., Rodrigue, M. M. D., de Carvalho, A. K. F., \& de Lima Soares, V. (2021). Sífilis Em Gestantes No Nordeste Do Brasil: Aspectos Epidemiológicos No Período DE 2010 E 2019. The Brazilian Journal of Infectious Diseases, $25,101324$.

Nonato, SM, Melo, APS, \& Guimarães, MDC (2015). Sífilis na gestação e fatores associados à sífilis congênita em Belo Horizonte-MG, 20102013. Epidemiologia e Serviços de Saúde, 24, 681-694.

Organização Mundial da Saúde. (2016). Diretrizes da OMS para o tratamento do Treponema pallidum (sífilis).

Pires, A. C. S., Oliveira, D. D., Rocha, G. M. N. M., \& Santos, A. (2014). Ocorrência de sífilis congênita e os principais fatores relacionados aos índices de transmissão da doença no Brasil da atualidade-revisão de literatura. Revista uningá review, 19(1).

Rocha, C. .C, Lima, T. S., Silva, R. A. N., \& Abrão, R. K. (2020). Abordagens sobre sífilis congênita. Research, Society and Development, 9 (8), e984986820-e984986820.

Rocha, A. F. B., Araújo, M. A. L., Barros, V. L. D., Américo, C. F., \& Silva Júnior, G. B. D. (2021). Complicações, manifestações clínicas da sífilis congênita e problemas relacionados à prevenção: revisão integrativa. Revista Brasileira de Enfermagem, 74.

Silva, M. J. N. D., Barreto, F. R., Costa, M. D. C. N., Carvalho, M. S. I. D., \& Teixeira, M. D. G. (2020). Distribuição da sífilis congênita no estado do Tocantins, 2007-2015. Epidemiologia e Serviços de Saúde, 29, e2018477.

Sousa, F. D. C. A., Lopes, D. S. D. C., da Costa, A. C. M., da Silva, E. B., da Silva, W. C., de Oliveira, E. H., \& Andrade, E. W. O. F. (2020). Perfil da Sífilis na Gestação no Período de 2007/2016 em Caxias-MA. Revista Enfermagem Atual In Derme, 91(29).

Sonda, E. C., Richter, F. F., Boschetti, G., Casasola, M. P., Krumel, C. F, \& Machado, C. P. H. (2013). Sífilis congênita: uma revisão da literatura. Revista de Epidemiologia e Controle de Infecção, 3 (1), 28-30.

Soares, K. K. S., Prado, T. N. D., Zandonade, E., Moreira-Silva, S. F., \& Miranda, A. E. (2020). Análise espacial da sífilis em gestantes e sífilis congênita no estado do Espírito Santo, 2011-2018. Epidemiologia e Serviços de Saúde, 29, e2018193. 